\title{
Correction to: Tracking physical activity in different settings from late childhood to early adulthood in Germany: the MoMo longitudinal study
}

Annette Rauner ${ }^{1 *}$, Darko Jekauc ${ }^{2}$, Filip Mess ${ }^{3}$, Steffen Schmidt ${ }^{1}$ and Alexander Woll ${ }^{1}$

\section{Correction}

After publication of the article [1], it has been brought to our attention that the values cited under "indices $\mathrm{t} 1-\mathrm{t} 0$ " have been reversed and should be displayed in the opposite order. The corrected Table 1 would appear as follows

Furthermore, the last sentence of Sport club physical activity (minutes per week) that reads "SCPA decreased by 39.3 min per week from to to t 1 in the young group ( $\mathrm{F} 177=15.4, \mathrm{df}=1, p<.001)$." should in fact say "SCPA increased by $39.3 \mathrm{~min}$ per week from to to t1 in the young group $(\mathrm{F} 177=15.4, \mathrm{df}=1, p<.001)$."

\footnotetext{
Author details

'Department of Sports and Sports Science, Karlsruhe Institute of Technology, Engler-Bunte Ring 15 (Building 40.40), 76131 Karlsruhe, Germany. 2Department of Sports Sciences, Humboldt-Universität zu Berlin, Philippstraße 13 (Building 11), 10111 Berlin, Germany. ${ }^{3}$ Institute of Health Sciences, University of Education Schwäbisch Gmünd, Oberbettringer Straße 200, 73525 Schwäbisch Gmünd, Germany.
}

Received: 18 January 2018 Accepted: 22 January 2018

Published online: 05 February 2018

\section{Reference}

1. Rauner A, Jekauc D, Mess F, Schmidt S, Woll A. Tracking physical activity in different settings from late childhood to early adulthood in Germany: the MoMo longitudinal study. BMC Public Health. 2015;15(1):391. https://doi.org/ 10.1186/s12889-015-1731-4.

\footnotetext{
* Correspondence: annette.rauner@kit.edu

'Department of Sports and Sports Science, Karlsruhe Institute of Technology, Engler-Bunte Ring 15 (Building 40.40), 76131 Karlsruhe, Germany

Full list of author information is available at the end of the article
} 
Table 1 Mean (1 standard deviation) patient characteristics $\left({ }^{*} p<.05\right)$

\begin{tabular}{|c|c|c|c|c|c|}
\hline & & LTPA (min./week) & SCPA (min./week) & OPA (days/week) & OS index (min./week) \\
\hline \multicolumn{6}{|c|}{ indices $-t_{0}$} \\
\hline overall & & $\begin{array}{l}74.1 \pm 98.0 \\
N=924\end{array}$ & $\begin{array}{l}218.6 \pm 122.8 \\
N=296\end{array}$ & $\begin{array}{l}3.47 \pm 1.82 \\
N=947\end{array}$ & $\begin{array}{l}292.7 \pm 164.5 \\
N=932\end{array}$ \\
\hline \multirow[t]{2}{*}{ sex } & boys & $\begin{array}{l}81.3 \pm 107.0 \\
N=438\end{array}$ & $\begin{array}{l}231.1 \pm 124.2 \\
N=168\end{array}$ & $\begin{array}{l}3.78 \pm 1.80 \\
N=447\end{array}$ & $\begin{array}{l}312.4 \pm 171.0 \\
N=438\end{array}$ \\
\hline & girls & $\begin{array}{l}67.3 \pm 88.1 \\
N=496\end{array}$ & $\begin{array}{l}200.5 \pm 118.6 \\
N=128\end{array}$ & $\begin{array}{l}3.15 \pm 1.78 \\
N=500\end{array}$ & $\begin{array}{l}267.8 \pm 151.2 \\
N=494\end{array}$ \\
\hline \multirow[t]{2}{*}{ age } & young group & $\begin{array}{l}86.4 \pm 104.5 \\
N=477\end{array}$ & $\begin{array}{l}193.6 \pm 101.6 \\
N=181\end{array}$ & $\begin{array}{l}3.82 \pm 1.75 \\
N=477\end{array}$ & $\begin{array}{l}280.0 \pm 145.3 \\
N=117\end{array}$ \\
\hline & old group & $\begin{array}{l}72.9 \pm 97.3 \\
N=469\end{array}$ & $\begin{array}{l}227.1 \pm 126.8 \\
N=130\end{array}$ & $\begin{array}{l}3.43 \pm 1.82 \\
N=469\end{array}$ & $\begin{array}{l}300.0 \pm 179.6 \\
N=815\end{array}$ \\
\hline \multirow[t]{4}{*}{ SES } & low & $\begin{array}{l}64.5 \pm 101.4 \\
N=185\end{array}$ & $\begin{array}{l}189.3 \pm 107.1 \\
N=39\end{array}$ & $\begin{array}{l}3.29 \pm 1.91 \\
N=186\end{array}$ & $\begin{array}{l}253.8 \pm 134.6 \\
N=184\end{array}$ \\
\hline & middle & $\begin{array}{l}81.9 \pm 100.6 \\
N=485\end{array}$ & $\begin{array}{l}224.6 \pm 120.7 \\
N=166\end{array}$ & $\begin{array}{l}3.70 \pm 1.76 \\
N=494\end{array}$ & $\begin{array}{l}306.5 \pm 170.6 \\
N=483\end{array}$ \\
\hline & high & $\begin{array}{l}69.6 \pm 89.8 \\
N=262\end{array}$ & $\begin{array}{l}222.0 \pm 133.1 \\
N=91\end{array}$ & $\begin{array}{l}3.15 \pm 1.78 \\
N=265\end{array}$ & $\begin{array}{l}291.6 \pm 158.6 \\
N=263\end{array}$ \\
\hline & \multicolumn{5}{|c|}{ indices $-t_{1}$} \\
\hline overall & & $74.1 \pm 93.5$ & $222.2 \pm 133.1$ & $3.31 \pm 1.86$ & $296.3 \pm 163.2$ \\
\hline \multirow[t]{2}{*}{ sex } & boys & $82.6 \pm 102.1$ & $250.5 \pm 132.9$ & $3.48 \pm 1.85$ & $333.1 \pm 165.3$ \\
\hline & girls & $64.2 \pm 83.5$ & $181.0 \pm 122.6$ & $3.15 \pm 1.87$ & $245.2 \pm 147.2$ \\
\hline \multirow[t]{2}{*}{ age } & young group & $58.6 \pm 87.2$ & $232.9 \pm 190.0$ & $3.20 \pm 1.79$ & $291.5 \pm 170.2$ \\
\hline & old group & $74.6 \pm 93.9$ & $228.9 \pm 138.8$ & $3.32 \pm 1.87$ & $303.5 \pm 162.3$ \\
\hline \multirow[t]{3}{*}{ SES } & low & $64.2 \pm 96.9$ & $230.0 \pm 109.3$ & $3.22 \pm 1.83$ & $294.2 \pm 146.4$ \\
\hline & middle & $73.3 \pm 89.4$ & $231.5 \pm 132.2$ & $3.30 \pm 1.88$ & $304.8 \pm 159.5$ \\
\hline & high & $82.2 \pm 97.0$ & $199.0 \pm 144.5$ & $3.38 \pm 1.88$ & $281.2 \pm 177.9$ \\
\hline \multicolumn{6}{|c|}{ indices $-t_{1}-t_{0}$} \\
\hline overall & & $0.0 \pm 128.5$ & $3.6 \pm 155.7$ & $-0.2 \pm 2.3^{*}$ & $3.6 \pm 192.2^{*}$ \\
\hline \multirow[t]{2}{*}{ sex } & boys & $1.3 \pm 141.6$ & $19.4 \pm 161.7^{*}$ & $-0.3 \pm 2.3^{*}$ & $20.7 \pm 206.4^{*}$ \\
\hline & girls & $-3.1 \pm 114.7$ & $-19.4 \pm 143.8^{*}$ & $0.0 \pm 2.3$ & $-22.6 \pm 167.7^{*}$ \\
\hline \multirow[t]{2}{*}{ age } & young group & $-27.9 \pm 139.0^{*}$ & $39.3 \pm 140.9^{*}$ & $-0.6 \pm 2.1^{*}$ & $10.5 \pm 183.0^{*}$ \\
\hline & old group & $1.7 \pm 127.2$ & $1.8 \pm 154.3$ & $-0.1 \pm 2.3$ & $3.5 \pm 192.9^{*}$ \\
\hline \multirow[t]{3}{*}{ SES } & low & $-0.3 \pm 138.5$ & $40.7 \pm 115.0^{*}$ & $-0.1 \pm 2.1$ & $40.4 \pm 176.7^{*}$ \\
\hline & middle & $-8.6 \pm 126.0$ & $6.0 \pm 151.1$ & $-0.4 \pm 2.4^{*}$ & $-1.7 \pm 186.8$ \\
\hline & high & $13.8 \pm 124.1^{*}$ & $-23.0 \pm 178.5^{*}$ & $0.2 \pm 2.3^{*}$ & $-10.4 \pm 202.3^{*}$ \\
\hline
\end{tabular}

SES - socioeconomic status; LTPA - leisure-time PA; SCPA - sport club PA; OPA - overall PA; OS index - overall sports index 